\title{
An Algebraic Approach to Temporal Logic
}

\author{
Burghard von Karger* \\ Institut für Informatik 2, Christian-Albrechts-Universität, Preusserstr. 1-9, D-24105 \\ Kiel, Germany. email: bvk@informatik.uni-kiel.d400.de
}

\begin{abstract}
The sequential calculus is an algebraic calculus, intended for reasoning about phenomena with a duration and their sequencing. It can be specialized to various domains used for reasoning about programs and systems, including Tarski's calculus of binary relations, Kleene's regular expressions, Hoare's CSP and Dijkstra's regularity calculus.

In this paper we use the sequential calculus as a tool for algebraizing temporal logics. We show that temporal operators are definable in terms of sequencing and we show how a specific logic may be selected by introducing additional axioms. All axioms of the complete proof system for discrete linear temporal logic (given in [9]) are obtained as theorems of sequential algebra.

Our work embeds temporal logic into an algebra naturally equipped with sequencing constructs, and in which recursion is definable. This could be a first step towards a design calculus for transforming temporal specifications by stepwise refinement into executable programs.

This paper is an extended abstract of a technical report [5] containing full proofs (available by $\mathbf{f t p}$ ). Most proofs have been omitted and simplifying assumptions were made to make the presentation easier.
\end{abstract}

\section{Introduction}

The nature and purpose of abstraction has been described by Dijkstra in [4].

We now take what is a standard step in mathematical theory building. The step is taken after the introduction of a notational novelty - such as a new abbreviation or a "mathematical macro" - for formulae that were interpreted in a familiar domain of discourse. The step consists of starting with a clean slate and axiomatising afresh the manipulations of the new formulae. In doing so, one creates a new domain of discourse; the rôle of the old, familiar domain of discourse, that used to constitute the subject matter, is thereby reduced to that of providing a possible model for the newly postulated theory. It is essential that the axioms of the new theory - which can be interpreted as theorems in the old universe of discourse - are clearly postulated as such and that the new theory is derived from them without any reference to the model of the

* This research has been funded by the Deutsche Forschungsgemeinschaft under grant KA $1052 / 1-1$ 
old universe of discourse. This is the only way to assure that the axioms of the new theory provide an interface that is independent of the old universe of discourse and that, hence, the new theory is safely applicable to alternative models.

Temporal logic is a perfect example. The "old domain of discourse" consists of sequences of states inside some computer and the notational novelty is a collection of temporal operators like $\bigcirc$ (next) and $\diamond$ (eventually). Abstraction is achieved by singling out a set of axioms from the set of laws governing the manipulation of temporal formulae.

Mathematics derives its power from the nesting of abstractions. Here is an example of such nesting. Peano's axioms abstract away from any particular representation of the set $\mathbf{N}$ of natural numbers. Further abstraction yields the concept of a semigroup. Semigroups are more general, and $\mathbf{N}$ is a very specific semigroup, namely it is infinite and cyclic. Conversely, every infinite and cyclic semigroup satisfies the Peano axioms.

Similarly, sequential algebra is an abstraction of temporal logic which is itself an abstraction of a concrete computational model. Sequential algebra is more general than temporal logic, and temporal logic can be characterized as a sequential algebra with specific properties.

The increase in abstraction is not of the trivial kind, like in "groups are more general than rings" which results from simply dropping some of the given structure. It is interesting because it is based on a radically different approach. As in the example, a logical characterisation (the Peano axioms) is replaced by algebraic structure (semigroups).

Abstractions usually enjoy the advantage of greater simplicity (semigroups are specified by just one axiom). A sequential algebra is just a complete boolean algebra with a semigroup structure and a few axioms giving the interaction between composition and the boolean connectives (whereas Manna and Pnueli's axiomatization of linear temporal logic has 17 axioms, not counting the boolean ones). Semigroups and boolean algebras with operators are thoroughly investigated subjects of algebra and the connection to temporal logic might enable valuable cross fertilization.

Abstraction frequently offers opportunities of building additional algebraic structure. Semigroups can, under certain conditions, be embedded into groups; in our example this yields a construction of the integers. Our work embeds temporal logic into an algebra that is naturally equipped with programming constructs like sequential composition, nondeterministic choice and conditionals, and in which recursion is definable.

It is hard to foresee all possible uses of a new abstraction, but let us list some objectives worth striving for.

- Power: The embedding mentioned in the previous paragraph could be a first step towards a design calculus for transforming temporal specification by stepwise refinement, where each step replaces some abstract subterm by a more concrete one, until an executable program is reached. 
- Taxonomy: Various temporal logics can be identified as subtheories of sequential algebra and large parts of sequential algebra theory can be developed before specialising to any particular of them. We should aim at a general theory, which can be specialised to interesting and/or well-known calculi or logics by adding axioms in an incremental fashion.

- Applications: In a pragmatic sense, the abstract approach may also help to discover new applications of temporal logic. The existing axiomatisation of temporal logic has been guided by a specific computational model, and the sheer number of axioms makes it hard to think of alternative models satisfying them. Showing that all of these axioms are consequences of a small number of assumptions expressible in a more abstract universe may make it easier to think of new situations where these assumptions also hold.

It is important to understand that sequential algebra is not proposed as an alternative to, or replacement of, temporal logic, in the same sense that semigroup theory cannot make Peano's axioms obsolete. Sequential algebra provides a very roundabout way of arriving at the simple and elegant set of operators of temporal logic. Their original discovery was a great intellectual achievement, and the value of temporal logic has been demonstrated abundantly in practical applications. Sequential algebra, on the other hand, aims at abstraction, integration, and classification, rather than direct usefulness for engineering problems. It is not decidable, and it is not complete. It cannot be, or else it could not encompass any theory that lacks these properties.

The rest of this paper is structured as follows. In the next section we gently introduce the sequential calculus. Section 3 collects basic concepts and laws, most of them familiar from the calculus of relations. Building on this, section 4 introduces the operators of temporal logic. In section 5 we come to the algebraic laws of temporal expressions, adding new axioms as needed on the way, until we obtain as theorems precisely the axioms listed in [9]. In the final section we discuss what progress we have achieved towards the three objectives set out above.

Except for a few examples, to show the style, we have to omit all proofs. They can be found in [5] (some also in [6]). But we do provide all the necessary lemmas, and the reader who was worked in the relational or a similar calculus should not find it too hard to fill the proofs in.

\section{The Sequential Calculus}

The relational calculus has been remarkably successful in reasoning about possibly non-deterministic systems, provided that their behaviour can be fully characterised by observation of just their initial and final states [1, 8, 12]. Many alternative models have been proposed for reactive systems, whose behaviour between initiation and termination is also of significance. A common feature of these calculi is that past observations cannot be cancelled or undone by what comes after. 
The general modelling technique is to represent each possible system as a set, whose elements represent single observations of a single experiment on the system described. In the relational calculus [13], the observations are pairs $(s, t)$, where $s$ and $t$ are drawn from the same set of states. In the calculus of intervals [3], these are required to be related by a total ordering $(s \leq t)$. In regular expressions [7] the observations are finite sequences of letters drawn from an alphabet $A$. In the regularity calculus [4], the sequences are given the structure of a free group. Another example is interval-based temporal logic (which is not to be confused with the point-based temporal logic studied in this paper), where observations are functions from time intervals to states, where time is a total ordering and may be discrete or continuous, finite or infinite $[10,14]$.

Any calculus for sets should start from consideration of the properties of their members; and sequential algebra relies only on qualities shared by all the observation spaces in question. The most basic common property is the existence of an associative composition operator $(x ; y)$, which makes a possibly longer observation from subobservations $x$ and $y$. For regular expressions, this is just concatenation of strings, and for free groups it is the group multiplication. In other cases, composition is a partial operator: in the relational calculus, the pair $(r, s)$ can be composed with $\left(s^{\prime}, t\right)$ only if $s=s^{\prime}$; and when this equality holds, the intermediate state is omitted:

$$
(r, s) ;(s, t)=(r, t) .
$$

Similarly, in interval temporal logic, composition is defined only when the end time and final state of the first operand are the same as the start time and initial state of the second operand. Then the two functions are compatible, so that their union is a function and can be taken as the result of the composition.

To help reasoning about the definedness of composition, we introduce two functions between observations. Each observation $x$ has a left unit $\overleftarrow{x}$ and a right unit $\vec{x}$, which satisfy the unit properties for composition:

$$
\overleftarrow{x} ; x=x=x ; \vec{x} .
$$

For example, in the relational and interval calculi

$$
\overleftarrow{(s, t)}=(s, s) \text { and } \overrightarrow{(s, t)}=(t, t)
$$

In interval temporal logic $\bar{x}$ is the initial state and time, whereas $\vec{x}$ is the final state and time. In both cases, composition is defined just when the right unit of the left operand is the same as the left unit of the right operand:

$$
x ; y \text { is defined iff } \vec{x}=\bar{y}
$$

In regular expressions (as in free groups) there is just a single unit for composition, the empty sequence. As a consequence $\rightarrow$ and $\leftarrow$ are constant functions, and composition is everywhere defined. 
The unit functions have two additional properties ${ }^{2}$ : they map units to themselves, and they depend only on the left or right operands of composition:

$$
\begin{array}{ccc}
\overleftarrow{x}=\vec{x}=\overleftarrow{x} & \text { and } & \overleftarrow{\vec{x}}=\overrightarrow{\vec{x}}=\vec{x} \\
\overleftarrow{x ; y}=\overleftarrow{x} & \text { and } & \overrightarrow{x ; y}=\vec{y} .
\end{array}
$$

Another property shared by all our models of reactive systems is cancellativity:

$$
\text { If } x ; y=x^{\prime} ; y^{\prime} \text { then }\left(x=x^{\prime}\right) \equiv\left(y=y^{\prime}\right) \text {. }
$$

To formalize the idea that time can only progress in a single dimension we introduce a prefix ordering on observations. If $x=y ; z$ then $y$ is said to be a prefix of $x$ (and $z$ a postfix). We postulate

If $x$ and $y$ are prefixes of $z$ then $x$ is a prefix of $y$, or $y$ is a prefix of $x$.

In reactive systems, it is not possible to backtrack or rewrite history by an inverting operation. The only action that can be undone is the trivial action that has not actually done anything, i.e. a unit $\overleftrightarrow{z}$. So we require that units are indivisible

$$
x ; y=\overleftarrow{z} \text { implies } x=y=\overleftarrow{z}
$$

The last assumption seems reasonable and it considerably simplifies the presentation of temporal logic through sequential algebra. However, we make it only for pedagogical reasons; the entire development is possible without it. It is given in full in [5].

Recall that the domain of temporal logic is given by

$$
I \stackrel{\text { def }}{=} \Sigma^{w} \times \mathbf{N}
$$

where $\Sigma$ is some given set of states. Every observation $(\sigma, n) \in I$ consists of an infinite sequence of states and one position in that sequence, and a temporal formula $P$ may be seen as denoting a set of them, namely

$$
\{(\sigma, n) \in I \mid(\sigma, n) \models P\} .
$$

But the observations $(\sigma, n)$ have no duration. The only natural way to impose an observation space structure on $I$ is by defining a trivial composition operator, namely $i ; i=i$ and $i ; j$ undefined for all distinct $i, j \in I$.

To make composition useful, we embed $I$ into a larger space $L$ in such a way that $I$ is precisely the set of all units of $L$. Instead of a single index $n$ into the sequence $\sigma$, we allow a pair $m \leq n$ of indices, specifying a (finite) interval, rather than a point. Composition of $(\sigma, m, n)$ and $\left(\sigma^{\prime}, m^{\prime}, n^{\prime}\right)$ is defined only when $\sigma=\sigma^{\prime}$ and $n=m^{\prime}$; and then we put

$$
(\sigma, m, n) ;\left(\sigma, n, n^{\prime}\right)=\left(\sigma, m, n^{\prime}\right) .
$$

2 These properties endow the observation space with the structure of a small category 
Identifying $(\sigma, n)$ with $(\sigma, n, n)$ we are lead to define the projection operators by

$$
\overleftarrow{(\sigma, m, n)}=(\sigma, m) \text { and } \overrightarrow{(\sigma, m, n)}=(\sigma, n)
$$

A characteristic of this observation space is that each observation carries within it a knowledge of the entire past and future. Consequently, two observations with the same, say, left unit can only be distinguished by their duration, and the longer one must be an extension of the shorter. Formally

$$
\begin{aligned}
& \overleftarrow{x}=\overleftarrow{y} \quad \Rightarrow \quad x \text { is a prefix of } y \text { or } y \text { is a prefix of } x \\
& \vec{x}=\vec{y} \quad \Rightarrow \quad x \text { is a postfix of } y \text { or } y \text { is a postfix of } x
\end{aligned}
$$

An observation space with these properties is called deterministic, because each state determines the entire past and future.

Now let $L$ be some fixed observation space. The objective of sequential algebra is to formalise a calculus of subsets of $L$. These obviously form a complete boolean algebra with join $U$, meet $\cap$, complement $\ulcorner$, least element $O$ and greatest element $L$. Just as relational composition is a lifted form of the composition of pairs (1), our more general sequential composition is obtained by lifting the composition defined for single observations.

$$
P Q \stackrel{\text { def }}{=}\{z \mid \exists x \in P, y \in Q . x ; y=z\} .
$$

The identity element of composition is

$$
I \stackrel{\text { def }}{=}\{x \mid \overleftarrow{x}=x=\vec{x}\} \text {. }
$$

The absence of a general converse forces us to define a relative converse $P / Q$, to play the same role that $P \breve{Q}$ plays in the relational calculus. Each observation of $P / Q$ is obtained from an observation of $P$ by cutting from the end something that is an observation of $Q$ :

$$
P / Q \stackrel{\text { def }}{=}\{x \mid \exists z \in P, y \in Q . x ; y=z\} .
$$

and, symmetrically,

$$
Q \backslash P \stackrel{\text { def }}{=}\{y \mid \exists x \in Q, z \in P . x ; y=z\} .
$$

We rule that composition binds more strongly than / and \, and, in turn, these bind more strongly than $U$ and $n$.

We now perform the abstraction step described by Dijkstra by listing a number of theorems valid in the power set algebra over an observation space and taking these as the axioms of a new algebraic structure, called a sequential algebra.

2.1 Definition A sequential algebra is a complete boolean algebra equipped with a constant $I$ and three binary operators $P Q, P \backslash Q$, and $P / Q$ satisfying axioms $2.2-2.5$. 
2.2 Monoid axiom Composition is associative and has $I$ as its identity.

The second axiom is the Schröder law from the relational calculus, except that $P \breve{Q}$ and $\breve{P} Q$ are replaced by $P / Q$ and $P \backslash Q$. The term 'exchange' is due to Dijkstra.

\subsection{Exchange axiom $P Q \subseteq \bar{R}$ iff $P \backslash R \subseteq \bar{Q}$ iff $R / Q \subseteq \bar{P}$.}

Not all laws of relational algebra translate into laws of sequential algebra. For example,

$$
(P Q) \breve{R}=P(Q \breve{R})=P(R \breve{Q})^{\cup}
$$

holds in relational algebra, whereas in sequential algebra no two of the expressions $(P Q) / R, P(Q / R)$ and $P /(R / Q)$ are equal. In fact, $P Q / R$ describes observations obtained from $P Q$ by cutting off the right something in $R$. So do the other two terms, but in a more specific way. In $P(Q / R)$ the cut has to be placed after the transition from $P$ to $Q$, whereas in $P /(R / Q)$ it must be before.

\subsection{Split axiom $\quad P Q / R=P(Q / R) \cup P /(R / Q)$.}

The last axiom of sequential algebra is the following

\subsection{Converse Axiom $\quad I / P=P \backslash I$.}

We do not explain this axiom here (but see [5]), because we will not use it. Our assumption that units are indivisible permits a much stronger axiom

\subsection{Indivisibility Axiom $\quad I / L=I$.}

We need not postulate the symmetric counterpart of the last axioms, because they can be proved as theorems. The sequential calculus enjoys a perfect symmetry between past and future and with every theorem we prove we get for free its time-wise dual.

An observation space is deterministic iff its power set algebra satisfies the following law

\subsection{Determinacy axiom $(P / L) L=P L \cup P / L$,}

To understand this, note that $P / L$ is the prefix closure of $P$. This axiom does not seem to imply its time-wise dual, so we postulate that also. Most of our theory can be developed without the determinacy axiom, and we will draw special attention to the place where it is needed.

This concludes the abstraction step. From now on observations must not be mentioned anymore (except for motivating definitions). Additional operators may only be defined in terms of the basic ones, and proofs must solely rely on the axioms of sequential algebra. 


\section{Basics of Sequential Algebra}

In this section we define the analogues of some basic concepts of the relational calculus, and list relevant algebraic laws. - The composition and both of the partial converse operators are universally disjunctive. In particular, they have $O$ as left and right zero. Moreover, the following laws hold

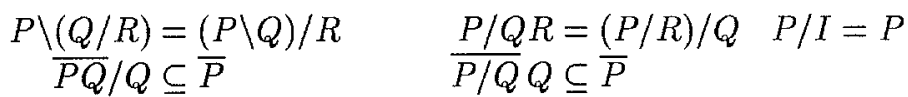

$$
\begin{aligned}
& (R \cap P / S) S \supseteq R S \cap P \quad(R \cap P S) / S \supseteq R / S \cap P
\end{aligned}
$$

Iteration is defined by $P^{0}=I, P^{i+1}=P P^{i}$, and $P^{*}=\bigcup_{i \geq 0} P^{2}$.

\subsection{Predicates}

In the relational calculus, a state $s$ can be represented by the observation $(s, s)$. So, a predicate on states may be represented as a set of observations $x$ with $\overleftarrow{x}=x=\vec{x}$. Generalising this idea to the sequential calculus, we call $P$ a predicate iff $P \subseteq I$. By convention, $B$ and $C$ always denote predicates. Let us define a negation operator by

$$
\neg P \stackrel{\text { def }}{=} \bar{P} \cap I .
$$

We allow logical notation for predicates

$$
\begin{array}{ccc}
B \vee C & \stackrel{\text { def }}{=} B \cup C & B \wedge C \stackrel{\text { def }}{=} B \cap C \\
\mathbf{T} \stackrel{\text { def }}{=} I & \mathbf{F} \stackrel{\text { def }}{=} O
\end{array}
$$

Clearly, the set of all predicates forms a boolean algebra. Here are some laws:

$$
\begin{aligned}
& B(P \cap Q)=B P \cap Q \quad B C=B \cap C \quad(B \cap C) P=B P \cap C P \\
& (\neg B) L=\overline{B L} \quad B P=B \backslash P \\
& P B / Q=P / Q B \quad B P / Q=B(P / Q) \quad P / B Q=(P / Q) B
\end{aligned}
$$

\subsection{Domain and Codomain}

As in the relational calculus, every $P$ can be projected to its domain or codomain

$$
\overleftarrow{P}=\{\overleftarrow{x} \mid x \in P\} \quad \text { and } \quad \vec{P}=\{\vec{x} \mid x \in P\}
$$

These definitions can be given at the calculus level by

$$
\overleftarrow{P} \stackrel{\text { def }}{=} I \cap P / P \text { and } \vec{P} \stackrel{\text { def }}{=} I \cap P \backslash P .
$$

They satisfy a couple of useful Galois connections.

$$
\begin{aligned}
(\overleftarrow{P} \subseteq B) & \equiv(P \subseteq B L) \\
(\overleftarrow{P B} \subseteq \neg C) & \equiv(\overrightarrow{C P} \subseteq \neg B)
\end{aligned}
$$


Here are some more laws

$$
\begin{array}{llll}
\overleftarrow{P} P=P & \overleftarrow{B}=B & L / P=L \overleftarrow{P} \\
\overleftarrow{B P}=B \overleftarrow{P} & \overleftarrow{P Q}=\overleftarrow{P Q} & \overleftarrow{P} L=(P / L) L
\end{array}
$$

The next law depends on the determinacy axiom. It is crucial for establishing that the next and previous operators cancel each other out.

$$
\overrightarrow{\bar{P} Q}=\overleftrightarrow{P \backslash Q} \cup \overleftarrow{Q \backslash P}
$$

The proof proceeds by indirect equality:

$$
\begin{aligned}
& \overrightarrow{\overline{P Q}} \subseteq \neg C \\
\equiv & \overleftarrow{Q C} \subseteq \neg \overleftarrow{P} \\
\equiv & Q C \subseteq(\neg \bar{P}) L \\
\equiv & Q C \subseteq \overline{\bar{P} L} \\
\equiv & Q C \subseteq \overline{P L \cup P / L} \\
\equiv & (Q / C \subseteq \overline{P L}) \wedge(P / L \subseteq \overline{Q C}) \\
\equiv & (P L C \subseteq \bar{Q}) \wedge(Q C L \subseteq \bar{P}) \\
\equiv & (P \backslash Q \subseteq \overline{L C}) \wedge(Q \backslash P \subseteq \overline{C L}) \\
\equiv & (P \backslash Q \subseteq L(\neg C)) \wedge(Q \backslash P \subseteq(\neg C) L) \\
\equiv & \overrightarrow{P \backslash Q} \cup \overleftarrow{Q \backslash P \subseteq \neg C}
\end{aligned}
$$

(by the Galois connection (6))

(by the Galois connection (7)) (since $(\neg B) L=\overline{B L}$ ) $(\overleftarrow{P} L=(P / L) L$, determinacy axiom $)$ $(Q C=Q / C$, boolean algebra $)$ (exchange axiom) (exchange axiom) (since $(\neg B) L=\overline{B L}$ ) (by the Galois connection (6))

\subsection{The All-the-Time Operator}

Suppose $x$ is an observation with the following property: Whereever you cut $x$ in two, you find a state at which the predicate $B$ holds:

$$
x=y ; z \quad \Rightarrow \quad \vec{y} \in B .
$$

Then $B$ is said to hold all the time in $x$. The set $[B]$ of all observations $x$ with this property can be defined as follows:

$$
[B] \stackrel{\text { def }}{=} \overline{L(\neg B) L} .
$$

The all-the-time operator is conjunctive and distributes over composition

$$
\begin{aligned}
{[B \cap C] } & =[B] \cap[C] \\
{[B] \cap P Q } & =([B] \cap P)([B] \cap Q) .
\end{aligned}
$$

Moreover, $B$ can be retrieved from $[B]$ by applying the domain (or codomain) operator

$$
\overleftarrow{[B]}=B=\overrightarrow{[B]} \text {. }
$$




\section{The Operators of Temporal Logic}

The smallest measurements of progress are non-unit observations that cannot be further decomposed into non-unit subobservations. Such observations correspond to single transitions of the observed system. The set of all transitions is described by

$$
\text { step } \stackrel{\text { def }}{=} \bar{I} \cap \overline{\bar{I}} \overline{\bar{I}} .
$$

$O B$ holds at some state $s$ iff there is a transition $x$ with $\overleftarrow{x}=s$ and $B$ holds at $\vec{x}$. The time-wise dual of the 'next' operator is the 'previous' operator $\ominus$.

$$
O B \stackrel{\text { def }}{=} \overleftarrow{\text { step } B} \quad \ominus B \stackrel{\text { def }}{=} \overrightarrow{B \text { step }}
$$

$B$ 'until' $C$ holds at a state $s$ if there is an observation $x$ with $\bar{x}=s$ such that $B \vee C$ holds all the time in $x$ and $C$ holds at the end. The time-wise dual is 'since':

$$
B \mathcal{U} C \stackrel{\text { def }}{=} \overleftarrow{[B \vee C] C} \quad B \mathcal{S} C \stackrel{\text { def }}{=} \overline{C[B \vee C]}
$$

The remaining operators are defined in terms of the four basic ones

$$
\begin{array}{lll}
\diamond B \stackrel{\text { def }}{=} \mathbf{T} \mathcal{U} B & \square B \stackrel{\text { def }}{=} \neg \diamond \neg B & B \mathcal{W} C \stackrel{\text { def }}{=} \square B \vee B \mathcal{U} C \\
\ominus B \stackrel{\text { def }}{=} \mathbf{T} \mathcal{S} B & \boxminus B \stackrel{\text { def }}{=} \neg \ominus \neg B & B \mathcal{B} C \stackrel{\text { def }}{=} \boxminus B \vee B \mathcal{S} C \\
\widetilde{\circlearrowleft} B \stackrel{\text { def }}{=} \neg \bigcirc \neg B & \widetilde{\Theta} B \stackrel{\text { def }}{=} \neg \ominus \neg B & \\
\text { first } \stackrel{\text { def }}{=} \neg \ominus \mathbf{T} & \text { last } \stackrel{\text { def }}{=} \neg \bigcirc \mathbf{T} &
\end{array}
$$

\section{The Algebra of Temporal Logic}

In this section we sketch how the axioms of a temporal logic may be proved as theorems of sequential algebra. The first task is to establish the (almost-Galois) connection between 'next' and 'previous':

$$
(B \subseteq \bigcirc C) \quad \text { iff } \quad(B \subseteq \neg l a s t) \wedge(\ominus B \subseteq C) .
$$

We prove the direction from right to left.

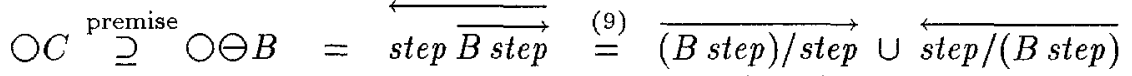

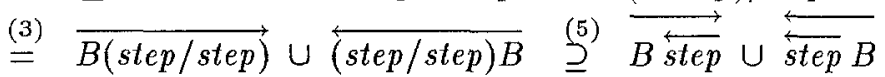

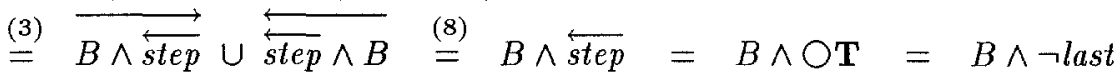

$$
\begin{aligned}
& \stackrel{\text { premise }}{\supseteq} B \text {. }
\end{aligned}
$$


- The definitions of the last section make also sense for continuous time (i.e. step $=O$ ), except that, of course, 'next' and 'previous' map everything to $\mathbf{F}$. We will now restrict ourselves to the discrete case. We postulate ${ }^{3}$.

\subsection{Discreteness axiom $\quad L=s t e p^{*}$.}

Under the assumption of discreteness, 'until' can also be defined in terms of iteration

$$
B \mathcal{U} C=\overleftarrow{(B \text { step })^{*} C}
$$

which, in turn, gives a more intuitive definition of 'eventually'

$$
\diamond B=\bigcup_{i \geq 0} \bigcirc^{i} B
$$

It is now easy to prove that the temporal operators satisfy the following algebraic laws

$$
\begin{aligned}
\widetilde{O} B & =\text { last } \vee \bigcirc B \\
\square B & \subseteq B \\
\bigcirc-B & \subseteq \neg B \\
\widetilde{O}(B \rightarrow C) & =\widetilde{O} B \rightarrow \widetilde{\bigcirc} C \\
\square(B \rightarrow C) & \subseteq \square B \rightarrow \square C \\
\square B & \subseteq \square \widetilde{\bigcirc} B \\
\text { first } \wedge \square B & \subseteq \square \widetilde{\Theta} B \\
\square(B \rightarrow \widetilde{O} B) & \subseteq \square(B \rightarrow \square B) \\
\text { first } \wedge(B \rightarrow \widetilde{\ominus} B) & \subseteq \square(B \rightarrow \boxminus B) \\
B \mathcal{W} C & =C \vee(B \wedge \widetilde{O}(B \mathcal{W} C)) \\
\square B & \subseteq B \mathcal{W} C \\
\text { first } & \subseteq \widetilde{\Theta} \mathbf{F} \\
B & \subseteq \widetilde{O} \ominus B
\end{aligned}
$$

We have not, so far, made any assumptions violating time-wise symmetry, so the temporal duals of the above laws also hold. But Manna and Pnueli's logic applies to computations that continue forever: no computation can ever reach a last state. So we postulate

${ }^{3}$ The discreteness axiom can be weakened so as to allow infinite sequences of discrete transitions. A full treatment is given in [5]. This is not needed for the observation space suggested as domain for temporal logic on page 2 , but is useful for dealing with recursion. 
5.2 Progress axiom last $=\mathbf{F}$. dual $\widetilde{O}$.

Since $\widetilde{\bigcirc} B=$ last $\vee \bigcirc B$, this implies that $\bigcirc$ coincides with its de Morgan

Our declared objective is to prove the axioms of temporal logic as theorems in sequential algebra. Since the conventions of algebra differ from those of logic we need to explain the correspondence.

In logic, temporal expressions achieve meaning through a validity relation, whereas in our approach they denote elements of a sequential algebra. Fortunately all we need to relate the two is a slight change of perspective.

Recall that a state corresponds to a pair $(\sigma, n)$ where $n$ is an index into the computation sequence $\sigma$; such a state is initial iff $n=0$. In the logic we aim at, a formula is said to be valid if it holds at all initial states. Translating to algebra, we define a predicate $B$ to be valid iff

$$
\text { first } \subseteq B
$$

Temporal logic has stronger versions of implication and equivalence, denoted by $\Rightarrow$ and $\Leftrightarrow$. They are defined by

$$
(B \Rightarrow C) \stackrel{\text { def }}{=} \square(B \rightarrow C)
$$

and

$$
(B \Leftrightarrow C) \stackrel{\text { def }}{=}(B \Rightarrow C) \wedge(C \Rightarrow B) .
$$

$B$ is said to entail $C$ iff $B \Rightarrow C$ is valid. Two predicates $B$ and $C$ that entail each other are congruent.

\subsection{Lemma Assume $B \subseteq C$. Then $B$ entails $C$.}

Using this lemma, and the equality of $\bigcirc$ and $\widetilde{O}$, we can translate the laws (11) into temporal validities.

5.4 Theorem All of the following predicates are valid

$$
\begin{array}{ll}
\square B \rightarrow B & \\
\bigcirc \neg B \Leftrightarrow \neg \bigcirc B & \ominus B \Rightarrow \widetilde{\ominus} B \\
\bigcirc(B \rightarrow C) \Leftrightarrow(\bigcirc B \rightarrow \bigcirc C) & \widetilde{\Theta}(B \rightarrow C) \Leftrightarrow(\widetilde{\ominus} B \rightarrow \widetilde{\ominus} C) \\
\square(B \rightarrow C) \Rightarrow(\square B \rightarrow \square C) & \boxminus(B \rightarrow C) \Rightarrow(\boxminus B \rightarrow \boxminus C) \\
\square B \rightarrow \square \bigcirc B & \square B \rightarrow \square \widetilde{\Theta} B \\
(B \Rightarrow \bigcirc B) \rightarrow(B \Rightarrow \square B) & (B \Rightarrow \widetilde{\ominus} B) \rightarrow(B \Rightarrow \boxminus B) \\
(B \mathcal{W} C) \Leftrightarrow(C \vee(B \wedge \bigcirc(B \mathcal{W} C))) & (B \mathcal{B} C) \Leftrightarrow(C \vee(B \wedge \bigcirc(B \mathcal{B} C))) \\
\square B \Rightarrow(B \mathcal{W} C) & \widetilde{\ominus} \mathbf{F} \\
B \Rightarrow \bigcirc \ominus B & B \Rightarrow \widetilde{\ominus} \bigcirc B .
\end{array}
$$


This is precisely the set of axioms given in [9]. - Manna and Pnueli define $\mathcal{U}$ and $\mathcal{S}$ in terms of $\mathcal{W}$ and $\mathcal{B}$, whereas we had to do it the other way round. This is not a problem, because their definitions come out as theorems

$$
B \mathcal{U} C=B \mathcal{W} C \wedge \diamond C \text { and } B \mathcal{S} C=B \mathcal{B} C \wedge \ominus C \text {. }
$$

Manna and Pnueli assume that computations do not extend infinitely into the past: every computation sequence $\sigma$ must have a first element. This is expressed by the following postulate:

\subsection{Well-foundedness axiom $\ominus$ first $=\mathbf{T}$.}

It is a curious fact that all axioms of temporal logic can be proved without invoking well-foundedness. Its only use is in proving the entailment theorem, which states that congruence in logic corresponds precisely to equality in algebra.

5.6 Entailment Theorem $B$ entails $C$ if and only if $B \subseteq C$.

Proof. $B$ entails $C$

$$
\begin{aligned}
& \equiv \text { first } \subseteq \square(B \rightarrow C) \\
& \equiv \diamond \neg(B \rightarrow C) \subseteq \neg \text { first } \\
& \equiv \forall i \geq 0: O^{i} \neg(B \rightarrow C) \subseteq \neg \text { first } \\
& \equiv \forall i \geq 0: \overleftarrow{\operatorname{step}^{i} \neg(B \rightarrow C)} \subseteq \neg \text { first } \\
& \equiv \forall i \geq 0: \overrightarrow{\text { first step }}{ }^{i} \subseteq(B \rightarrow C) \\
& \equiv \forall i \geq 0: \Theta^{i} \text { first } \subseteq(B \rightarrow C) \\
& \equiv \ominus \text { first } \subseteq(B \rightarrow C) \\
& \equiv B \subseteq C
\end{aligned}
$$

(by def. of entailment and validity)

(by def. of $\square$ )

(by (10))

(by def. of $\bigcirc$ and $\stackrel{\leftarrow}{P Q}=\overleftarrow{P Q}$ )

(since $(\overleftrightarrow{P B} \subseteq \neg C) \equiv(\overrightarrow{C P} \subseteq \neg B)$ )

(see two steps above)

(see four steps above)

(by well-foundedness)

\section{Conclusion}

We showed that all axioms of Manna and Pnueli's logic correspond to theorems of deterministic sequential algebra. Conversely, as we have explained in section 2 , the domain $\Sigma^{\omega} \times \mathbf{N}$ of this logic can be embedded into an observation space, and then our definitions of $\bigcirc$ etc. coincides with the standard one. From this it follows that every equation between temporal expressions that can be proved in sequential algebra gives a valid congruence of temporal logic.

To summarise, we have modelled Manna and Pnueli's logic precisely. We have proved all the axioms as theorems, and we have shown that no theorem can be derived that would correspond to an invalid formula.

In the introduction we suggested three aims of an algebraic approach to temporal logic. Let us now review how much progress has been made towards these objectives. 
Power. We have not attempted to outline a design calculus here, but a first attempt has been made in a companion paper [2]. But the proofs we have given (and those we had to omit) illustrate the interplay between the temporal expressions that pertain to single points and the sequential constructs which enable us to calculate with longer sections of a computation. They are also very similar in flavour to the relational calculus, which has been so successful for design and correctness proofs of conventional programs. This makes us hope that the sequential calculus may be similarly efficacious for the construction of reactive processes.

There is another interesting possibility. We considered the temporal operators as operators on predicates only, but nothing in their definitions prevents us from applying them to arbitrary arguments. For example, the predicate $O(x:=x+1)$ holds at some point iff the next but one transition increments $x$ by one.

In another paper [6], we developed a fragment of interval temporal logic from sequential algebra (without needing any additional axioms). So we have also integrated point-based and interval-based temporal logic in a single framework, simpler and more general than either.

Taxonomy. We developed one variety of temporal logic from sequential algebra by adding four axioms to sequential algebra, each of which corresponds to one of four orthogonal design decisions (of Manna and Pnueli): The determinacy axiom for linearity of time, the discreteness axiom, the progress axiom for allowing only infinite computation sequences, and the well-foundedness axiom for requiring that every computation sequence must have a first element. Other choices lead to different points in temporal logic country, but it will need someone more widely travelled than this author to attempt a complete mapping.

We have also seen that definitions can be taken and many theorems can be proved before additional axioms are introduced. In particular, practically all work can be done before breaking time-wise symmetry. Late commitments are the key to avoiding painful duplication and backtracking.

Applications. Observation spaces provide models for sequential algebra, thereby providing a way of finding new uses for temporal logic. A promising candidate is Hoare's theory of Communicating Sequential Processes. Observation spaces for CSP are well explored [11] and all we need is to add a little twist to make them deterministic. It remains to be seen if this integration of CSP with relational calculus and temporal logic can lead to improved design methods for concurrent programs.

\section{Acknowledgements}

Thanks to Tony Hoare for supervising and encouraging the emergence of the sequential calculus, and for many helpful comments on an earlier version of this paper. 


\section{References}

1. R. Berghammer, P. Kempf, G. Schmidt, and T. Ströhlein. Relation algebra and logic of programs. In Algebraic Logic, volume 54 of Colloquia Mathematica Societatis János Bolyai. Budapest University, 1988.

2. R. Berghammer and B. von Karger. Formal derivation of CSP programs from formal specifications. submitted to MPC 95, 1995.

3. S. M. Brien. A time-interval calculus. In R. Bird, C. Morgan, and J. Woodcock, editors, Mathematics of Program Construction, LNCS 669. Springer-Verlag, 1992.

4. E. W. Dijkstra. The unification of three calculi. In M. Broy, editor, Program Design Calculi, pages 197-231. Springer Verlag, 1993.

5. B. v. Karger. Temporal logic as a sequential calculus. Procos technical report [kiel bvk 17], Christian-Albrechts-Univ., Inst. f. Inf. und Prakt. Math., Kiel, 1994. available via WWW, http://wrw.informatik.uni-kiel.de/ procos/kiel.html .

6. B. v. Karger and C.A.R. Hoare. Sequential calculus. To appear in Information Processing Letters, 1995.

7. S. Kleene. Representation of events in nerve nets and finite automata. In Shannon and McCarthy, editors, Automata Studies, pages 3-42. Princeton University Press, 1956.

8. R. Maddux. A working relational model: The derivation of the Dijkstra-Scholten predicate transformer semantics from Tarski's axioms of the Peirce-Schröder calculus of relations. Manuscript, 1992.

9. Z. Manna and A. Pnueli. The Temporal logic of Reactive and Concurrent Systems-Specification. Springer-Verlag, 1991.

10. B. Moszkowski. Some very compositional temporal properties. Technical Report TR-466, University of Newcastle, 1993. Accepted for Procomet 1994, San Miniato.

11. E. Olderog and C. Hoare. Specification oriented semantics for communicating processes. Acta Inf., 23:9-66, 1986.

12. G. Schmidt and T.Ströhlein. Relations and Graphs. EATCS Monographs on Theoretical Computer Science. Springer, 1991.

13. A. Tarski. On the calculus of relations. Journal of Symbolic Logic, 6(3):73-89, 1941.

14. C. Zhou, C.A.R. Hoare, and A. P. Ravn. A calculus of durations. Information Processing Letters, 40:269-276, 1992. 